Cuidados paliativos y medicina intensiva en la atención al final de la vida del siglo XXI

\title{
Palliative care and Intensive Medicine in health care at the end of life in the $X X I$ century
}

\author{
P. Loncán ${ }^{1}$, A. Gisbert ${ }^{2}$, C. Fernández ${ }^{3}$, R. Valentín ${ }^{4}$, A. Teixidó ${ }^{5}$, R. Vidaurreta ${ }^{6}$, \\ I. Saralegui ${ }^{7}$
}

\section{RESUMEN}

El objetivo fundamental de la Medicina Intensiva es la atención al paciente crítico, es decir, personas con una enfermedad o condición amenazante para su vida pero con posibilidades de recuperación; a pesar de los tratamientos, incluidos los de soporte vital, con frecuencia no se consigue la curación y es entonces cuando se plantea la retirada de los mismos por criterios de futilidad. Una vez adoptada esa decisión los esfuerzos deben dirigirse a aplicar los cuidados necesarios para conseguir un final de la vida sin dolor ni sufrimiento, procurando que el paciente esté acompañado de sus seres queridos. Estudios realizados muestran las lagunas formativas y asistenciales que existen en la atención a los pacientes en el final de la vida dentro del ámbito de las Unidades de Medicina Intensiva. El presente artículo revisa la aportación que los Cuidados Paliativos pueden ofrecer para mejorar la asistencia a los enfermos que fallecen en UMI y a sus familiares. La ontología de cuidados paliativos tiene como objetivos la mejoría sintomática, la prevención de las posibles complicaciones, conocer y respetar los criterios de bienestar del paciente y la atención a la familia en el proceso final y en el duelo. Por tanto, implica a diversas disciplinas del campo de la salud y las impelen a trabajar con objetivos comunes. En situaciones de final de la vida el conocimiento y la sensibilidad de los profesionales tienen que estar al servicio del compromiso de fidelidad con el paciente que permita reorientar los esfuerzos terapéuticos hacia los objetivos propuestos.

Palabras clave. Medicina Intensiva. Cuidados Paliativos. Final de la vida. Reorientación esfuerzo terapéutico.

\begin{abstract}
The basic aim of Intensive Care Medicine is care for the critical patient, that is, persons with a disease or condition that is life-threatening but with possibilities of recovery. In spite of the treatments, even those involving life support, cure is frequently not achieved and this is when the question is raised of withdrawing treatment due to criteria of futility. Once that decision is taken, efforts must be directed towards applying the necessary care to achieve an end to life without pain or suffering, endeavouring to ensure that the patient is accompanied by his loved ones. Studies show the shortcomings in training and health care that exist in caring for patients at the end of their lives within the sphere of the Intensive Care Units. This article reviews the contribution that can be made by Palliative Care in improving care for patients that die in the ICU and for their relatives. The ontology of palliative care aims to achieve an improvement of symptoms, the prevention of possible complications, to determine and respect the welfare of the patient, and to help the family in the final process and in mourning. It therefore involves different disciplines from the health field which must work with common aims. In situations of the end of life the knowledge and sensitivity of the professionals must be at the service of faithful commitment to the patient, making it possible to redirect therapeutic efforts towards the proposed aims.
\end{abstract}

Key words. Intensive Care Medicine. Palliative Care. End of life. Redirection of therapeutic efforts.
Grupo de Bioética de la SEMICYUC. Grupo de Bioética de la SECPAL.

1. Unidad de Cuidados Paliativos. Fundació Residència Sta Susanna. Caldes de Montbui. Barcelona.

2. Unidad Hospitalización domiciliaria. Hospital Clínico. Valencia.

3. Atención Primaria do Servizo Galego de Saúde. Lugo.

4. Equipo de Cuidados Paliativos.Cáceres.

5. Unidad Cuidados Paliativos. Hospital St Jaume. Calella. Barcelona.

6. Unidad de Cuidados Paliativos y Daño cerebral irreversible. Fundación Instituto S. José. OHSJD. Madrid.

7. Unidad de Medicina Intensiva. Hospital Santiago Apóstol. Vitoria.

\section{Correspondencia:}

Pilar Locán

Unidad de Cuidados Paliativos

Fundació Residència Sta Susanna

Caldes de Montbui. Barcelona

e-mail: ploncan@fundaciostasusanna.com 


\section{EL FINAL DE LA VIDA DE LOS PACIENTES EN UNA UNIDAD DE MEDICINA INTENSIVA (UMI)}

El ingreso de un paciente en la UMI pretende revertir o paliar su situación crítica, con una atención sanitaria de calidad que procure el restablecimiento de la salud, el alivio del dolor y del sufrimiento. A pesar de emplear los tratamientos adecuados para cada patología, debido a la gravedad del tipo de paciente atendido en las UMI, se produce una mortalidad superior a la de otros servicios hospitalarios. Siendo el fallecimiento de un paciente un fenómeno frecuente en las UMI (en Estados Unidos el $20 \%$ de las muertes se producen en la $\mathrm{UMI}^{1}$ ), estudios como el SUPPORT ${ }^{2}$ (A Controlled Trial to Improve Care for Seriously Ill hospitalizad patients: The Study to Understand Prognoses and Preferences for Outcomes and Risks of Treatments) pusieron en evidencia que el tratamiento al final de la vida de los pacientes críticos y la atención a las necesidades de sus familiares están lejos de ser adecuados. No siempre se proporcionan los cuidados y procedimientos para conseguir una muerte sin sufrimiento, de acuerdo a las preferencias del paciente y coherente con la práctica clínica y valores culturales. Entre las causas principales destaca la insuficiente formación en medicina paliativa de los profesionales sanitarios, incluyendo las habilidades de comunicación ${ }^{3}$.

\section{CUIDADOS PALIATIVOS}

El término "paliativo" deriva de pallium, palabra latina que significa "capa", capote; etimológicamente significa proporcionar una capa para calentar a "los que pasan frío", una vez que no pueden ser curados. Los cuidados paliativos se centran en el alivio del dolor y del sufrimiento en los pacientes que están en la fase final de su vida, sea cual sea la causa o enfermedad inicial. En 1990 la OMS definió cuidados paliativos como "el cuidado activo total de los pacientes cuya enfermedad no responde ya al tratamiento". Tiene prioridad el control del dolor, de otros síntomas y problemas de orden psicológico, social y espiritual. El objetivo de los cuidados paliativos es pro- porcionar la mejor calidad de la vida para los pacientes y sus familiares ${ }^{4}$.

El marco conceptual de los Cuidados Paliativos está fundamentado en las propuestas de trabajo del Hastings Center ${ }^{5}$, en tanto que promueve una asistencia a las personas en fases de enfermedad avanzada para evitar la obstinación terapéutica y el abandono del paciente cuando su curación no es posible. Este marco incorpora a los logros de la medicina en su lucha contra la enfermedad, la atención al sufrimiento cuando la muerte está próxima y la ayuda a morir en paz, tal y como también proclama la Declaración de la Asamblea parlamentaria del Consejo de Europa, sobre los derechos de los enfermos terminales y moribundos ${ }^{6}$ y la Declaración sobre la atención médica al final de la vida, de la Organización Médica Colegial y la Sociedad Española de Cuidados Paliativos ${ }^{7}$. Según la definición de la OMS la atención activa, global y holista va dirigida tanto a las personas que sufren una enfermedad avanzada, progresiva e incurable como a su entorno afectivo ${ }^{8}$. La ontología de cuidados paliativos tiene como objetivos la mejoría sintomática y la prevención de las posibles complicaciones, conocer los criterios de bienestar del paciente y la atención a la familia en el proceso final y en el duelo. Por tanto, implica a diversas disciplinas del campo de la salud y las impelen a trabajar con objetivos comunes.

\section{EVOLUCIÓN HISTÓRICA DE LA ÉTICA DE LOS CUIDADOS PALIATIVOS}

La ética de los cuidados paliativos no es distinta de la de otros ámbitos de la asistencia sanitaria, pero se ha de aplicar en contextos de máxima fragilidad, así como de prudencia en la toma de decisiones, en una práctica clínica deliberativa y responsable.

Los cuidados paliativos se iniciaron en la década de los sesenta en los países anglosajones con el movimiento hospice. Inicialmente el objetivo era conseguir que los pacientes dispusiesen de los días que les quedasen conscientes y libres de dolor, con los síntomas controlados, de manera que estuviesen con dignidad, en su casa o 
en un lugar similar, rodeados de la su entorno más cálido.

Twycross considera que el movimiento hospice tiene sus orígenes en los Hospicios medievales del S. XIX, y posteriormente en los Hospice católicos de Dublín y Londres. Cecily Saunders es la persona que fundó el St. Chistopher's Hospice considerado como la cuna de los cuidados paliativos ${ }^{9}$. Por tanto, en sus inicios los cuidados paliativos han estado muy influidos por las creencias religiosas de las personas que los pusieron en marcha. De hecho su integración en el sistema sanitario público ha sido difícil y actualmente los Hospice funcionan como centros concertados con la Sanidad Pública ${ }^{10}$.

Los cuidados paliativos se desarrollaron posteriormente en Estados Unidos, gracias a la colaboración de la psiquiatra suiza, residente en Estados Unidos Elisabeth Kübler Ross ${ }^{11}$. A partir de aquí, se desplegaron programas de Cuidados paliativos en Canadá y en diferentes países europeos. En España comienzan a abrirse a partir del año 1984, en Santander y Barcelona. Desde entonces han ido aumentando aunque de manera irregular en todo el territorio. En este contexto, las tres últimas décadas han aportado a la visión de los cuidados paliativos la multiculturalidad, como coexistencia de diferentes estructuras sociales y como confluencia de valores $^{12}$. La mayor esperanza de vida hace coexistir modelos ideológicos y de familia más distantes, aumentando las diferencias intergeneracionales.

El modelo ético que durante muchos años tuvo una mayor vigencia, desde el inicio del movimiento hospice, fue el de la ética de la convicción, relacionado tradicionalmente con la ética del cuidado y la ética de los cuidados de enfermería. Se trata de una ética que concede valor moral a los fines pero no a los medios. Es, por tanto, una ética heterónoma. Cecily Saunders, fundadora del movimiento hospice inició su actividad como enfermera, y aunque su mentalidad debía estar más próxima a la revolución moral y asistencial de Nightingale $^{13}$, tenía algunos rasgos típicos de la ética de la convicción. Existía también la creencia, en los primeros años de los cuidados paliativos, de que unos cuidados paliativos correctos solo podían hacerse desde unas convicciones religiosas profundas ${ }^{14}$. Otros autores han destacado lo que llaman la "agenda oculta" de algunos profesionales que trabajan en cuidados paliativos, ya que sus iniciativas han estado inspiradas por motivos religio$\operatorname{sos}^{15}$. La confusión existente entre los cuidados paliativos y la lucha contra la eutanasia es otro rasgo de la ética de la convicción, expresado en los inicios del movimiento hospice. Posteriormente la influencia de Norteamérica a favor de los derechos civiles, incrementó el respeto hacia las decisiones autónomas de los pacientes.

La tesis de Diego Gracia en su articulo: "Ética de los Cuidados paliativos: entre la convicción y la responsabilidad"16, es que los cuidados paliativos han ido evolucionando en los últimos años hacía una ética de la responsabilidad, considerando a ésta como la más adecuada para elaborar una ética que integre el respeto a los principios y las consecuencias previsibles, permitiendo así tomar decisiones respetuosas, razonables y prudentes. Max Weber acuñó el término de la ética de la responsabilidad en los inicios del siglo XX y trató de dar un valor moral a la realización de los fines a través de los medios disponibles. Es decir, equilibrar principios y consecuencias, lo que Aristóteles denominaba phrónesis (prudencia).

Podemos afirmar que la bioética desde sus inicios es una ética de la responsabilidad que asume el respeto a todos los seres humanos como un absoluto, en el sentido de que todos tienen derecho a participar en un proceso libre y equitativo de deliberación.

El modelo propuesto por Pilar Arranz y $\operatorname{cols}^{17}$ sobre las estrategias comunicativas como mediaciones para la deliberación moral es un buen modelo de ética de la responsabilidad. Roy y McDonald también consideran que la tarea de la ética en cuidados paliativos consiste en elaborar juicios prácticos que tengan en cuenta pacientes concretos y situaciones únicas. Por tanto, nos obliga a un ejercicio continuo de responsabilidad $^{18}$. La comprensión de la ética 
del cuidado recibió un gran impulso con el análisis feminista, especialmente la visión de Carol Gilligan ${ }^{19}$, una ética de la virtud en el sentido de arethé o hábito, en la que no es posible el distanciamiento afectivo a que daría lugar una ética de la convicción.

Uno de los problemas más importantes de los cuidados paliativos ha sido y sigue siendo delimitar con claridad en qué consiste el bien específico que prestan a la sociedad, ya que cuando se introducen conceptos valorativos como confort o dignidad, en muchas ocasiones contienen una gran carga de subjetividad. La relación médico-enfermo solo puede estar basada en una relación de confianza y debe de intentar llegar a un modelo deliberativo de relación en la que el médico trata de respetar la autonomía del enfermo y de conocer sus valores iniciando con él una deliberación conjunta para obtener una solución óptima o menos lesiva ${ }^{20}$.

Concluyendo, la tendencia actual es a considerar la relación médico-enfermo como un proceso de negociación entre personas que se respetan mutuamente en su autonomía en el que el modelo deliberativo es el fundamento ético del mismo ${ }^{21}$. Ello no implica un "relativismo moral", sino la comprensión de los seres humanos como integrantes de una red de dependencias, en la que el cuidado es actitud y práctica efectiva imprescindible.

\section{CONSIDERACIONES SOBRE EL MODELO DE ATENCIÓN DE LOS CUIDADOS PALIATIVOS}

Tanto en los cuidados paliativos generales, es decir, aquellos que se prestan en todos los niveles de asistencia, como en los cuidados paliativos avanzados, que por su complejidad requieren equipos específicos el modelo de atención se basa en: a) una actitud con empatía hacia la percepción subjetiva que el paciente y su familia tiene de su realidad, tan substancial como un dato clínico es la apreciación del enfermo cuando hablamos de su bienestar; b) una comunicación efectiva y la construcción de una relación de compromiso que implican cualidades humanísticas, habilidades en comunicación y disponibilidad de tiempo y espacio adecuado para cada paciente; c) aceptación de la muerte como el final natural de la vida tratando de aliviar la desesperación y el sufrimiento.

El modelo de atención al final de la vida ha de estar dirigido a la atención al sufrimiento y a la muerte y estar basado en la persona (respetando la intimidad y confidencialidad), garantizar el respeto a la autonomía práctica, contextualizar el proceso de la enfermedad (proporcionalidad de las acciones, utilidad de las intervenciones), avalar las decisiones (responsabilidad más allá del acto) y ser sensible con el entorno afectivo del paciente. La forma de aplicarlo implica elaborar un plan de cuidados "con el paciente" de forma anticipada, siempre que sea posible ${ }^{22}$ o según las voluntades expresadas. Sobre las bases ético-clínicas de la situación concreta se planifican las actuaciones de acuerdo con las expectativas del paciente y las disponibilidades técnicas. Las condiciones técnicas necesarias para garantizar las consideraciones mencionadas incluyen aspectos:

Estructurales. Tanto la información al paciente y/o la familia como la toma de decisiones precisan de un grado de intimidad que hacen necesarias estructuras físicas que lo permitan. También hay que garantizar el tiempo suficiente de los profesionales para poder establecer el dialogo y llevar a cabo una comunicación efectiva.

Formativos. Resulta básica la destreza en el manejo del control sintomático y la capacitación de los profesionales en aspectos como la comunicación, la evaluación de la competencia de los pacientes y la toma de decisiones ético-clínicas.

Metodológicos. A nivel organizativo el funcionamiento de los equipos asistenciales se debe basar en un trabajo multi e interdisciplinario, que contemple la existencia de espacios para reuniones en las que se intercambie la información que permita elaborar el plan terapéutico individualizado y consensuado. Por otra parte los instrumentos de evaluación (sintomáticos, cualitativos, etc.) han de estar adaptados y ser aplicados según las posibilidades emocionales y de tiempo de los enfermos. La conexión entre los servicios internos hospitalarios y unidades funcionales, así como con los comunitarios es necesaria. 
Tanto en el medio hospitalario como en el domiciliario hay que asegurar la respuesta consensuada de los diferentes niveles asistenciales y la coordinación de los diferentes servicios sanitarios.

Documentales. Hay que orientar los modelos de historia clínica de forma que contemplen la recogida de datos sobre los aspectos relevantes (control síntomas, grado de competencia de los pacientes, registro de voluntades anticipadas). Disponer de Guías y/o Protocolos sobre las situaciones que con más frecuencia pueden presentar contradicciones $^{23}$ (Insuficiencia de órganos avanzada, Enfermedades degenerativas muy evolucionadas etc.), sobre la limitación del esfuerzo terapéutico $\mathrm{y} / \mathrm{o}$ parada de tratamientos ${ }^{24}$. Resultan recomendables protocolos de atención al paciente en fase de final de vida y sobre aspectos de la sedación ${ }^{25}$; para la obtención y/o consulta de las voluntades anticipadas del paciente y de soporte a la familia incluido el de atención al duelo.

\section{QUÉ PUEDE APORTAR EL MODELO DE CUIDADOS PALIATIVOS}

1. Valoración global de la situación del enfermo además de la específicamente orgánica.

2. Abordaje integral de la persona enferma.

3. Establecer comunicación con la familia desde el primer período.

4. Reorientar los esfuerzos terapéuticos en establecer medidas de confort de acuerdo con el pronóstico.

5. Elaborar un Plan Anticipado de Cuidados que permita valorar las voluntades anticipadas, las preferencias expresadas o conocidas.

6. Favorecer la reubicación del paciente en los recursos más adecuados.

7. Constatar que la atención facilitada concuerda con las expectativas del paciente y entorno afectivo, favorece el confort y da soporte cuando la curación no es posible.

8. Satisfacción al equipo por dar la mejor atención posible cuando la muerte está próxima.

\section{CONSIDERACIONES PRÁCTICAS ACERCA DE LA CALIDAD EN LA ATENCIÓN PALIATIVA}

Existen numerosas publicaciones sobre las posibilidades de evaluar la calidad asistencial a los enfermos. Más difícil resulta consensuar qué ha de ser considerado indicativo de dicha calidad ${ }^{26,27}$. Una reflexión desde la experiencia asistencial sobre qué ha de contemplarse cuando hablamos de calidad en un enfermo al final de la vida incluye aspectos físicos, de reconocimiento personal, afectivos-sociales y de trascendencia vital, que de forma descriptiva se especifica en:

\section{Buen tratamiento sintomático.}

2. Conocer como percibe el paciente su enfermedad y qué siente como amenazas subjetivas.

3. Establecer un pacto terapéutico basado en la confianza y respeto.

4. Facilitar las relaciones afectivas y dar soporte al entorno afectivo

5. Atender a la expresión de sentimientos sobre el sentido de la vida.

El paciente paliativo se caracteriza por la presencia de múltiples síntomas, multicausales y con componente multidimensional. Cualquiera de estos síntomas o molestias que les produce la enfermedad avanzada, van a estar modulados por diversos factores (tratamiento farmacológico, medidas generales, aspectos emocionales, sociales, económicos, espirituales, etc.). El paciente va a percibir de forma particular este síntoma (impacto) y es lo que va a expresar. Esta expresión es lo que como profesionales vamos a registrar y evaluar. Esto condiciona, por ejemplo, que cuando un paciente manifiesta que tiene dolor, no sólo se refiere a dolor físico, sino que la percepción dolorosa va a estar influenciada por sus vivencias previas, cultura, estado de ánimo, ansiedad, nivel cognitivo, estado de consciencia, etc.

Por otra parte, la familia se convierte también en objeto de tratamiento. Son dadores y receptores de cuidados. Son coprotagonistas. Sufren por y con el paciente. 
En estas circunstancias se hace necesaria la intervención de un equipo multidisciplinar que actúe de manera interdisciplinaria.

Chapman y Gravin $^{28}$, definieron sufrimiento como "complejo estado afectivo, cognitivo y negativo caracterizado por la sensación que tiene el individuo de sentirse amenazado en su integridad, el sentimiento de impotencia para hacer frente a dicha amenaza y por el agotamiento de recursos personales y psicosociales que le permitirían hacer frente a dicha amenaza". Teniendo en cuenta esta definición, en los pacientes paliativos se produce el denominado "triángulo del sufrimiento ", donde no sólo sufre el paciente, sino también la familia y el equipo de profesionales.

En relación al sufrimiento del paciente, va a estar determinado principalmente por cuatro grandes aspectos:

1. Síntomas físicos: dolor, xerostomía, disnea, estreñimiento, ...

2. Síntomas neuropsquiátricos : insomnio, ansiedad, depresión, delirium,...

3. Distrés existencial: desesperanza, pérdida de rol laboral y familiar, pérdida de significado vital, alteraciones de la imagen corporal,...

4. Distrés sociofamiliar: ver sufrir a sus seres queridos, sentirse una carga,...

Por este motivo la instauración de un plan terapéutico global, desde un punto de vista médico, va a ir encaminado a conseguir los siguientes objetivos:

1. Mejorar la calidad de vida

2. Promoción de la autonomía del paciente

3. Mejora de la adaptación emocional tanto del paciente como de la familia.

Para lograrlo se precisa de una adecuada evaluación, monitorización y control de síntomas, habilidades de comunicación e información, soporte psicosocial, así como de metodología de análisis de cuestiones éticas y de trabajo interdisciplinar. La función del médico y de enfermería es fundamental. Los profesionales no deben tener únicamente una preparación fundamentada en conocimientos científicos que exige el avance tecnológico moderno, ni tampoco pueden contentarse con ser solamente técnicos-científicos, sino que han de ser personas que ejerciten todos los días la sensibilidad ante el sufrimiento humano. Por ello la relación profesional de la salud-enfermo, es una relación eminentemente humana y entre sus deberes está crear un ambiente en el que los valores, costumbres y creencias del individuo sean respetados y ayudar al enfermo a mantener, desarrollar o adquirir autonomía personal, autorrespeto y autodeterminación, guiándonos siempre por criterios profesionales ${ }^{29}$.

El control sintomático requiere conocimientos sobre terapéuticas, sobre los instrumentos de valoración, utilizarlos de forma individualizada y con un seguimiento muy continuado. Ya que una de las cuestiones a tener presente respecto a los síntomas es la versatilidad en su percepción por parte de los pacientes (un mismo síntoma puede ser percibido de forma distinta según las personas y/o circunstancias) y el cambio en el tiempo de los mismos (en pocas horas puede variar) y habrá que estar alerta para adecuar el tratamiento. Estar abiertos a ofrecer explicaciones sobre el mismo, tanto al paciente como a la familia, facilita la relación clínica, favorece el cumplimiento y ayuda a cuidar a su entorno.

Sin duda enfermería es una profesión vocacional y cercana al paciente, y llega a establecer unos lazos estrechos, compartiendo momentos muy intensos y delicados con el paciente y sus familiares ${ }^{30}$. Entre sus objetivos están: proporcionar confort y preservar la calidad de vida, planificando cuidados adecuados en cada momento e individualizados que garanticen el mayor bienestar posible del paciente y siempre respetando su dignidad ${ }^{31}$. Para ello hay que preservar los horarios de descanso, modificar actividades de cuidados, instaurar medidas de confort en la higiene de piel y mucosas, procurar un ambiente tranquilo, observar en el paciente signos verbales y no verbales de dolor, agitación o inquietud. Como medidas de apoyo emocional se debe tratar al paciente con la misma actitud de respeto de siempre, mantener en todo momento la comunicación, sin olvidar la no verbal, como tocarle, cogerle la 
mano, hablarle cuando se realice algún cuidado. Respecto a la familia el objetivo como profesionales, es el proporcionar el apoyo y soporte durante el proceso y ofrecer la información adecuada en cada momento, referente al cambio de objetivos en el cuidado del enfermo como puede ser la suspensión de la alimentación, las curas no agresivas, la evolución del proceso y como pueden ayudar en el cuidado. Sabemos que un familiar que ha participado en el cuidado vivirá la pérdida de una forma más natural.

El cuidado postmortem hay que situarlo en el contexto del proceso, siendo importante incluir éste a la hora de la muerte, así como facilitar la certificación y proporcionar apoyo religioso y espiritual poniéndoles en contacto con el oficiante si así lo demandan o desean. Ofrecer apoyo, por si tienen dudas y mostrar una actitud abierta, amistosa y profesional.

\section{SOBRE EL SOPORTE PSICOLÓGICO}

El malestar que provocan los síntomas, los cambios en la vida, en la organización personal y familiar, el temor a la incapacidad o a la muerte...son algunas de las inquietudes que pueden estar en la mente del enfermo. En un proceso de atención global tenemos en cuenta esta posibilidad $\mathrm{y}$ atendemos a las manifestaciones particulares de cada uno de los pacientes. Además, éstos tienden a interpretar su estado en relación al modo de intervenir y de hablar de las personas que les cuidan. Los profesionales de cuidados paliativos aceptan el reto de atenderlos, sin embargo hacerlo no siempre es fácil. Por ello se ha promovido la presencia de psicólogos en los equipos de Cuidados Paliativos con la doble función de atender directamente a los casos que requieren atención especializada pero también para ofrecer soporte y formación a los profesionales en aquellas cuestiones de las que se pueden hacer cargo ellos mismos.

Las principales preocupaciones de los pacientes suelen ser debidas al dolor, al deterioro y la dependencia, a la falta de control, al temor de ser una carga, a la incertidumbre, al temor a morir, al temor a la soledad. Se tratará de tomar como problemas aquello que cada paciente considere como tal y no introducir nuevas cuestiones a no ser que esté muy justificado. Se tratará de ayudarle a buscar las soluciones o acompañarle en la falta de las mismas y en la toma de decisiones que él considere más adecuadas no imponiéndole las nuestras ${ }^{32}$.

En ocasiones también se revelan dificultades para dotar de sentido a la propia vida. Si la falta de sentido aparece en los enfermos terminales es porque ante ellos se abre uno de los enigmas más profundos de la vida humana: la muerte. En esos momentos las posiciones personales serán diversas y es importante no confundir espiritualidad con religión. Para algunos puede ser urgente encontrar una respuesta que cancele esa hiancia, la religión suele ser un buen recurso porque ofrece un sentido preestablecido. La falta de sentido también se cancela mediante el amor, es por esto que observamos que se necesita más de lo habitual la presencia de los seres amados. En algunas ocasiones y si cuentan con un psicoanalista que les acompañe los pacientes prefieren enfrentarse a ese vacío y revisar las identificaciones que les han sostenido para encontrar ahora una nueva y mejor respuesta.

Lo más importante es detectar en cada caso cual es el modo en que el paciente podrá afrontar la situación y así poder adaptar nuestros recursos. Para ello es imprescindible escuchar sin prejuicios lo que él dirá.

También hay que respetar su silencio. Hay pacientes que prefieren no hablar porque de ese modo pueden ignorar temores que no podrían superar.

Los recursos que las personas pueden movilizar tienen que ver con la estructura de su personalidad, con el tipo de problema de que se trate, con sus experiencias previas, el modo de resolver conflictos habitualmente y también depende de las respuestas que reciben por parte de los profesionales $^{33}$. En ocasiones no es preciso el soporte especializado y sólo será necesario orientar las intervenciones del equipo. En otros casos será necesario que el profesional de la psicología se haga cargo del caso. En este contexto es adecuado el uso de criterios de derivación específicos 
que eviten un infradiagnóstico e infratratamiento, así como una atención dirigida sobre problemas específicos, intervención de forma precoz, evitar sobrecarga profesional y favorecer la formación y el lenguaje común entre profesionales ${ }^{34}$.

Respecto de los familiares, sus principales preocupaciones tienen que ver con los sentimientos de impotencia -por la inexistencia de soluciones para algunos problemas- y de culpa debidos a los sentimientos contradictorios que se pueden experimentar. También se atienden los procesos de duelo de los familiares. Al hacerse cargo de los afectos de los pacientes y sus allegados, no se puede considerar resuelto un caso sin una elaboración final que comprenda la disposición del equipo para acoger el vacío del doliente y ayudarle en el proceso inicial del duelo.

En conclusión, en los equipos de cuidados paliativos las decisiones se toman no solo en función de aquello que es posible hacer sino en función de los deseos e intereses de los implicados. Por este mismo motivo, porque se cuenta explícitamente con los pacientes y sus familiares, se conversa con ellos -distinguimos hablar a los pacientes de conversar con ellos-. Se suele argumentar que conversar con los pacientes requiere tiempo. Cierto, pero se trata de una inversión para la relación posterior. Si en un primer abordaje asumimos los problemas subjetivos, de paciente y familia, sentamos las bases de la confianza en la relación profesional.

Atender a los problemas psicológicos que plantean la enfermedad grave y la posibilidad de la muerte humaniza la medicina ${ }^{35}$, especialmente a cada uno de los implicados.

\section{ACERCA DEL SOPORTE SOCIAL}

"Los servicios y recursos sociales no son un acto de gracia, sino la satisfacción de un derecho". Partiendo de esta máxima podemos afirmar que el trabajador social es el profesional que hace de nexo de unión entre las necesidades de la persona y las instituciones existentes. Entre sus estrategias están explicar los recursos sociales, humanos y materiales existentes; transmitir a los familiares la seguridad de que se está haciendo lo mejor para cuidar al enfermo y tratar de fomentar el contacto familiar, social y atender los sentimientos espirituales o creencias que tenga el enfermo y la familia.

El Trabajador Social tiene la obligación tanto profesional como ética en Cuidados Paliativos de intentar mejorar, paliar y ayudar sobre el entorno, la familia, el paciente y el equipo. Por encima del utilitarismo social los Trabajadores Sociales deben tender, en Cuidados Paliativos, a no valorar únicamente los recursos y el reparto más igualitario de estos, si no tender más a humanizar la asistencia tanto a los pacientes como la familia, dada su situación de final de vida, valorando las necesidades particulares de cada caso.

En definitiva, las posibilidades reales de perjudicar al paciente tienen un alcance infinito no solo desde el punto de vista clínico si no también social. Actualmente las personas han asumido un mayor protagonismo en sus cuidados siendo más participativas, más críticas y haciendo valer sus derechos. Los trabajadores sociales tienen el deber ético de apoyarles y velar por ellas cuando no puedan hacerlo por si mismas. Los cambios de estilo de la relación, en el fondo y en la forma, han provocado a los profesionales la tentación de aplicar recursos ilimitados, en cuya prevención desarrolla un papel defensivo la Ética, creando la obligación de aplicar bien los recursos sociales sin discriminar. Los profesionales sanitarios y todos los que tienen a su cuidado personas, están obligados a hacer el bien, es decir, a promover acciones que beneficien tanto a sus enfermos como a sus personas de apoyo (sus cuidadores). Esto implica que todos los profesionales pongan sus conocimientos, habilidades y valores al servicio de los demás.

\section{CUIDADOS PALIATIVOS: REALIDADES Y METAS}

Los cuidados paliativos han crecido, de manera considerable a nivel mundial. En nuestro país existen actualmente casi 300 equipos específicos de cuidados paliativos distribuidos entre atención hospitalaria, domiciliaria, equipos consultores hospitalarios y hospitales de día ${ }^{36}$. 
El reto está en garantizar la accesibilidad a los cuidados paliativos a todos los pacientes que las precisen, con independencia del diagnóstico, en cualquier lugar, circunstancia o situación dentro de las diferentes estructuras de la red sanitaria ${ }^{37}$. Sería recomendable introducir el modelo de atención en el momento del diagnóstico de enfermedad que limita las expectativas de vida de forma próxima. El Plan Estratégico $^{38}$ del Ministerio de Sanidad y Consumo, que define los cuidados paliativos como un derecho del ciudadano, plantea un modelo colaborador, interdisciplinario basado en la coordinación entre niveles asistenciales y entre recursos convencionales y específicos de cuidados paliativos, incorporando la recomendación de optimizar la formación en todos los niveles del sistema de salud. Queda implícito que el sistema sanitario requiere una transición del modelo multidisciplinario al interdisciplinario para poder abordar con garantías la atención paliativa.

En el análisis de la atención paliativa en nuestra sociedad se detecta: una relación beneficios/coste buena con una disminución de visitas a urgencias; camas menos costosas y posibilidad de morir en casa; una valoración de la calidad asistencial alta; trato profesional específico; información adecuada y con respeto a los criterios del paciente; la evolución de los equipos específicos ha procurado experiencia y competencia. En definitiva, los servicios de cuidados paliativos mejoran la efectividad y eficiencia en la atención a los enfermos avanzados y terminales ${ }^{39}$. Los aspectos a mejorar son a nivel organizativo una cobertura insuficiente, una accesibilidad variable y un despliegue incompleto ${ }^{40}$. Las propuestas de progreso tienen que contemplar la extensión de la atención a enfermos no oncológicos; ampliar la intervención en ámbitos específicos como servicios de urgencias, residencias geriátricas, unidades de cuidados intensivos, asistencia primaria; incluir la atención paliativa en los criterios de calidad de los hospitales de agudos.

Para ello resulta ineludible: la sensibilización de planificadores y gestores; el desarrollo de políticas integrales; la colaboración con las sociedades científicas que avalen criterios de intervención y metodologías de trabajo propias del modelo de atención de cuidados paliativos y promuevan los cambios de actitudes de los profesionales en el ámbito sanitario.

En este sentido, la colaboración de profesionales de los cuidados paliativos y de la medicina intensiva nos ha llevado a considerar de forma conjunta las cuestiones a tener presentes en la atención al final de la vida en las unidades de medicina intensiva.

\section{ASPECTOS ÉTICOS DE LOS CUIDADOS PALIATIVOS}

Una actitud de empatía de los profesionales con el sufrimiento resulta inexcusable para el acompañamiento al final de la vida. Asimismo, individualizar las intervenciones y reconocer que la variabilidad emocional interpersonal, en la vivencia ante la muerte, constituyen el entramado de la atención paliativa.

Las cuestiones éticas implicadas en cuidados paliativos se basan en el reconocimiento de que en el paciente incurable o en fase final no debe prolongarse la vida de forma innecesaria. Aquí reside una de las primeras dificultades: hacer una pausa en la atención cotidiana del enfermo para reevaluar la situación clínica, discutir con el resto del equipo sanitario (médicos y personal de enfermería) el pronóstico y plantear otras opciones de tratamiento valorando las posibilidades de respuesta al mismo. Ciertamente en la mayoría de los casos esta valoración es subjetiva aunque basada en parámetros y medidas como las escalas de gravedad o predictores de mortalidad, que aportan una estimación general, basada en series de casos y estadísticas, pero que no tiene en cuenta aspectos tan relevantes como la experiencia de los profesionales, la respuesta individual al tratamiento, las ganas de vivir del paciente o su apoyo familiar. Teniendo en cuenta estas consideraciones, los médicos tienen que reconocer los límites de la medicina y no aplicar tratamientos considerados fúti$\operatorname{les}^{41}$, entendiendo como tales aquéllos que no consiguen el objetivo que se esperaba de ellos. En este sentido, mantener tratamientos fútiles se considera mala práctica 
clínica $^{43}$ por ir en contra de la dignidad humana; por otro lado, consumir recursos sanitarios inútiles va en contra del principio de justicia distributivai.

Cuando el enfermo es considerado incurable se debe plantear la limitación de Tratamientos de Soporte Vital (LTSV), bien sea no iniciando nuevos tratamientos (withold) o retirando los ya aplicados (withdraw), estableciendo un consenso entre el equipo sanitario y los familiares o allegados del paciente ${ }^{44}$. En la mayoría de los casos el paciente es incompetente para tomar decisiones debido a su enfermedad o bien porque recibe fármacos sedantes; por ello la información y el acuerdo se centra en la familia, respetando sus preferencias si las ha expresado previamente de forma verbal o escrita a través de un Documento de Instrucciones Previas o Voluntades Anticipadas ${ }^{45}$, o teniendo en cuenta lo que el paciente hubiera elegido para sí mismo (juicio sustitutivo). Si bien, las implicaciones éticas de la limitación del esfuerzo terapéutico son las mismas para las acciones de no iniciar intervenciones como para las de detener o dejar de aplicar las mismas hay que reconocer la vulnerabilidad de los propios profesionales y que emocionalmente quizás es más difícil detener medidas interventoras que no iniciarlas. Compartir las decisiones éticas con otros profesionales del mismo equipo y consultar a otros expertos nos puede ayudar a alcanzar la excelencia asistencial (Unidades Funcionales Interdisciplinarias, Programas Atención Domiciliario). También hay que dejar constancia escrita en la historia clínica de cualquier decisión y acción con el fin de asegurar su cumplimiento.

En ocasiones la situación clínica puede hacer necesario la utilización de medidas como la sedación. La cuestión ética que plantea el tema de la sedación paliativa gira entorno al objetivo y al proceso en la toma de decisión de aplicar la sedación. La sedación paliativa se aplica en situaciones de final de vida con síntomas refractarios que causan sufrimiento, con el objetivo de disminuir el nivel de conciencia y con el consentimiento del paciente y/o su representante. La participación del paciente, en su defecto de su representante, o el cono- cimiento contrastado de sus preferencias en la toma de decisiones sobre la utilización de esta medida terapéutica, son condiciones básicas que han de quedar reflejadas en la historia clínica.

La relación clínica en los cuidados paliativos y en medicina intensiva se caracteriza por la trascendencia de las decisiones y por la fragilidad del paciente y de su familia. Por ello, el dilema -a menudo conflicto- entre autonomía y paternalismo están siempre en su fondo. Los profesionales sanitarios deben respetar la autonomía del paciente, aportando la información necesaria para la toma de decisiones, tener en cuenta sus preferencias y respetar la posibilidad de no aceptar un tratamiento propuesto. El rechazo del tratamiento no tiene que influir en la calidad de los cuidados paliativos; como expresan los anglosajones, no reanimar (DNR = Do not resucitate) no significa relajarse o abandonar al paciente (DNR $=D o$ not relax).

Se trata de reorientar el objetivo del tratamiento, el paciente no debe ser abandonado ya que a partir de ese momento necesita unos cuidados específicos, de calidad y proporcionados por personal sanitario formado para ello. Nos encontramos en la transición de la medicina intensiva con el fin de curar, hacia los cuidados paliativos, cuyo objetivo es el alivio de los síntomas, que se prolongarán allá donde se encuentre el paciente, hasta su fallecimiento. Velar por la coordinación con otros niveles y/o profesionales con el fin de garantizar la continuidad asistencial significa establecer una cadena ética. Cualquier decisión ética implica responsabilidad sobre el proceso y hay que establecer un plan de cuidados de compromiso con una atención adecuada y de acuerdo a las decisiones tomadas.

Los pasos a seguir para indicar cuidados paliativos en UMI

\section{Reconocer el final}

El primer paso es reconocer que el paciente no responde a los tratamientos aplicados y probablemente evoluciona al fallecimiento (active dying), en base a la experiencia clínica del equipo sanitario y a 
los parámetros y escalas de gravedad o predicción de mortalidad empleadas en cada caso. A partir de ese momento debe plantearse la LTSV, teniendo como objetivo el alivio de los síntomas para conseguir que el paciente esté lo más cómodo posible. Es el momento de cambiar el objetivo, pasar de curar a cuidar ${ }^{46}$.

Para facilitar la identificación precoz de los pacientes que evolucionan al fallecimiento puede ser útil la evaluación diaria de la situación clínica ${ }^{47}$, sobre todo en situaciones como las siguientes:

- Diagnóstico de una enfermedad o condición de pronóstico muy grave.

- Pobre calidad de vida previa a su ingreso

- Más de 5 días con ventilación mecánica.

- Más de 7 días de estancia en UMI.

La deliberación en las sesiones clínicas debe servir para definir los objetivos del tratamiento, con el respeto de las preferencias del paciente o sus familiares en caso de incompetencia. Se trata de evitar actitudes heroicas o tratamientos desproporcionados, escuchar la opinión de todos, incluida la del personal de enfermería, dando más importancia a la del médico responsable del enfermo por su conocimiento más preciso de la evolución clínica del paciente y del sentir de los familiares.

2. Reorientar el objetivo: alivio de los síntomas

La mayoría de los pacientes ingresados en UMI, y más aún aquellos que se encuentran en el final de su vida, tienen dolor y disnea $^{48}$. Otros síntomas que refieren habitualmente son: fatiga, náusea, tos, estreñimiento, ansiedad, depresión, delirio o insomnio. Por lo tanto los esfuerzos deben dirigirse a identificar ${ }^{49}$ la presencia de los mismos, utilizando las medidas y escalas apropiadas $^{50}$, para tratarlos adecuadamente.

\section{Información y búsqueda de acuerdos}

La comunicación entre el paciente, sus familiares o allegados y el equipo sanitario es uno de los pilares de la atención en el final de la vida. Las personas que acompa- ñan a sus parientes en los últimos momentos de su vida se encuentran en un medio desconocido para ellos ${ }^{51}$ y por ello los sanitarios tienen que procurar que esta situación sea lo más llevadera posible ${ }^{52}$. La mejor forma es fomentar la comunicación entre médicos, enfermeras, paciente y familiares. En las encuestas realizadas a familiares de pacientes ingresados en UMI, la queja más citada es la deficiente comunicación con el equipo sanitario, fundamentalmente con los médicos ${ }^{53}$.

Para mejorar la comunicación es recomendable cierta flexibilidad horaria, tanto para la información como para el tiempo dedicado a las visitas ${ }^{54}$. Si lo permite la estructura de la unidad, se procurará que puedan acompañar al enfermo en su propia habitación el mayor tiempo posible. Aunque médicos y enfermeras pueden sentir que los familiares junto a la cama del enfermo dificultan su quehacer, hay que reconocer que la inconveniencia para los sanitarios es mínima comparada con el sufrimiento de la familia ${ }^{55}$.

Además de facilitar el encuentro de los familiares con el enfermo, y más aún en los últimos momentos de vida, es aconsejable proporcionar asistencia religiosa si ésta es requerida, indicar los pasos a seguir en caso de óbito y reconfortarlos en la medida de lo posible ${ }^{56,57}$.

\section{Formularios, Protocolos y Registros}

Es aconsejable diseñar formularios que recojan las órdenes médicas y cuidados de enfermería específicos para pacientes en el final de la vida atendidos en UMI, y que pueden ser empleados en el caso de su traslado a otro servicio hospitalario ${ }^{58}$. Permite unificar la práctica, evitar confusiones y disminuir los errores de tratamiento, además de facilitar la evaluación posterior para realizar una adecuado control de calidad.

5. Involucrar a la familia en el plan de cuidados

Los profesionales deben intentar que los familiares sientan que son importantes y necesarios en la atención al final de la vida de su ser querido; es posible que ese papel activo en el cuidado pudiera minimi- 
zar el duelo por su pérdida ${ }^{59}$. Al mismo tiempo que se le da soporte emocional.

\section{Formación e integración de los Cuidados} Paliativos en UMI.

Teniendo en cuenta la frecuencia con la que se asiste a los pacientes en el final de su vida, la competencia en cuidados paliativos debiera ser parte de la formación en medicina intensiva ${ }^{60}$. El paciente precisa en esos momentos una asistencia multidisciplinar basada en:

- Alivio del sufrimiento físico.

- Alivio del sufrimiento psicológico, incluyendo los aspectos sociales y espirituales.

- Centrado en el paciente y su familia, procurando decisiones compartidas y respetando en lo posible sus preferencias.

- Coordinación con otros servicios implicados en la asistencia.

- Apoyo del personal sanitario que proporciona los cuidados paliativos.

Una de las recomendaciones para incorporar los contenidos del final de la vida en UMI puede ser la interconsulta con un servicio de Cuidados Paliativos, como se realiza en centros como Detroit Receiving Hospital o University of Rochester Medical Center. Aceptar la colaboración del personal experto puede ser el primer paso para integrar la medicina paliativa en el entorno de la $\mathrm{UMI}^{61}$. Hay que tener en cuenta que las UMI tienen unas características que hacen que el cuidado al final de la vida pueda ser más apropiado: relación enfermera/paciente baja, acceso i/v en la mayoría de los pacientes y estar acostumbrados a emplear sedoanalgesia. Por ello es posible conseguir una colaboración eficaz con el consejo del personal especializado en la atención al paciente que va a fallecer ${ }^{62}$.

Prestar asistencia a pacientes en el final de la vida y consolar a los familiares es una tarea que precisa una adecuada formación. Esta área de conocimiento apenas tiene espacio en el programa curricular de las facultades de medicina y escuelas de enfermería, así como en la formación post$\operatorname{grado}^{63}$. Para ello es necesario realizar pro- gramas formativos como el proyecto EPEC (Education on Palliative and End-of-lifecare) ${ }^{64}$.

\section{Procedimientos: sedación en el final de} la vida

El objetivo de la sedación en este contexto es el alivio del sufrimiento del paciente y no pretende acelerar la muer$\mathrm{te}^{65}$. Aplicar los cuidados y tratamientos adecuados para calmar el dolor, la agitación, la ansiedad o la disnea ${ }^{66}$, aunque con ellos se pueda adelantar el fallecimiento del enfermo en fase final, se ajusta a las recomendaciones éticas y al marco legislativo actual, y por lo tanto, es considerado como buena práctica clínica.

Existen dos tipos de sedación aplicada en pacientes a los que se limitan o retiran TSV:

Sedación paliativa: administración de fármacos en las dosis y combinaciones requeridas, para reducir la consciencia con el fin de aliviar uno o más síntomas refractarios, con el consentimiento explícito o delegado del paciente. La sedación paliativa no tiene porqué ser irreversible y puede ser necesaria en diversas situaciones clínicas.

Sedación terminal o sedación en la agonía: administración de fármacos para lograr el alivio de un sufrimiento físico y/o psicológico inalcanzable con otras medidas, mediante la disminución suficientemente profunda y previsiblemente irreversible de la consciencia, en un paciente cuya muerte se prevé muy próxima. Requiere el consentimiento explícito o delegado del mismo. En la mayoría de los pacientes críticos, debido a su incapacidad, este consentimiento se obtendrá del familiar o representante.

La sedación terminal persigue mitigar el sufrimiento del enfermo asumiendo, por "el principio del doble efecto", el efecto no deseado de la privación de consciencia hasta que ocurre la muerte. El fallecimiento será una consecuencia inexorable de la evolución de la enfermedad y/o sus complicaciones, no de la sedación. Si el alivio del dolor es difícil, lo es también en el entorno de las UMI por los problemas de comunicación, la gravedad de la enferme- 
dad, un nivel de consciencia disminuido y la inexactitud de los signos clínicos ${ }^{67}$. El empleo de escalas para la evaluación del dolor en pacientes conscientes son de utilidad a la hora de pautar la analgesia y sedación por objetivos y que sea fácilmente modificable por el personal de enfermería $^{68,69}$

Debe evitarse todo aquello que provoque incomodidad o malestar al paciente, para apoyar el tratamiento farmacológico con opioides, benzodiacepinas o neurolépticos, fundamentalmente. Las dosis deben individualizarse en función de la situación del paciente (edad, exposición previa, enfermedad subyacente, fracasos orgánicos, etc.) y los efectos perseguidos. No existen otros límites en la dosificación que los marcados por los objetivos propuestos, que deben estar claramente registrados en la historia clínica ${ }^{70}$, con el fin de orientar a todos los profesionales que participan en el cuidado del paciente y evitar malas interpretaciones y problemas de índole legal.

\section{Apoyo a los cuidadores (caring for care- givers)}

Los médicos y enfermeras que atienden a los pacientes en el final de la vida y a sus familiares, están sometidos a una carga de trabajo, fundamentalmente en el ámbito emocional, que en ocasiones les puede afectar personalmente. Con frecuencia necesitan apoyo por parte de sus compañeros, siendo necesaria la colaboración institucional para el adecuado desempeño de esta tarea ${ }^{71}$.

\section{RECOMENDACIONES FINALES}

La calidad de la atención sanitaria a los pacientes que fallecen en las UMI tras la limitación de tratamientos de soporte vital y el apoyo a sus familiares implica la prestación de unos cuidados paliativos adecuados. La formación de los profesionales de medicina intensiva a menudo es deficiente en éste área y por ello es necesaria una colaboración con las unidades de cuidados paliativos, además de incluir el aprendizaje de cuidados paliativos en la formación de los especialistas en pacientes críticos. Mejorar las habilidades para la comunicación con el paciente y sus familiares, discutir y registrar adecuadamente las órdenes de limitación de tratamientos, incluir la sedación y otros cuidados que procuren la comodidad del paciente, así como la evaluación posterior del proceso son la base de una atención integral y de calidad de las personas que no podemos curar pero a las que tenemos la obligación de cuidar.

\section{BIBLIOGRAFÍA}

1. Angus DC, Barnato AE, Linde-Zwirble WT, WEISSFELD LA, WATSON RS, RICKERT T et al. Use of intensive care at the end of life in the United States: An epidemiologic study. Critical Care Medicine 2004; 32: 638-643.

2. The SUPPORT principal investigators for the SUPPORT project. A controlled trial to improve care for seriously ill hospi talized patients; the Study to Understand Prognosis and Preferences for Outcomes and risk of Treatment. JAMA 1995; 274: 1591-1598.

3. NELSON J. Identifying and overcoming the barriers to high-quality palliative care in the intensive care unit. Crit Care Med 2006; 34: S324-S331.

4. World Health Organization. Cancer Pain Relief and Palliative: a report of a OMS expert committee. Geneva: OMS; 1990.

5. The Hastings Center Report. The Goals of Medicine: Setting New Priorities NovemberDecember, 1996.

6. Asamblea Parlamentaria del Consejo de Europa. Recomendación 1418 (1999) Protección de los derechos humanos y la dignidad de los enfermos terminales y moribundos. Adoptada el 25 de junio de 1999.. Disponible en http://www.unav.es/cdb /acoerec99-1418.html Visitada el 5 de octubre de 2007.

7. Ciprés L, Fernández JP, Gómez X,Gómez M, Pascual A, Pérez M, Porta J, Viñas J. Declaración sobre la atención médica al final de la vida. Med Pal 2002;9 (1): 30-31. Disponible en http://www.aeds.org/ documentos/dec_amfv.htm. Visitada el 5 de octubre de 2007.

8. The Hastings Center. Guidelines on the terminationof LIFE-Sustaining Treatment and the care of Dying. Bloomington Indiana: University Press, 1987.

9. Twycross RG. Hospice care, redressing the balance in medicine Journal of the Royal Society of Medicine, 1980; 73: 475-481. 
10. Hockley J. The evolution of the Hospice approach. En: Clark D, Hockley j, Ahmedzai $\mathrm{S}$ (eds). New themes in Palliative Care. Buckingham: Open University Press; 1997, 84-100.

11. KüBLER Ross E. On death and dying. Nueva York: Ed Mac Millan, 1969.

12. Blank R, Merrick JC: End of life decision making. A crosss-national study. MIT Press ISBN J-262-02574-4.

13. Nightingale F. Notes on nursing. Nueva Cork, Dover publications 1969; 25.

14. Clark D. Cicely Saunders: Founder of the Hospice Movement. Selected letters 19591999. OxFord, OxFord University PRESS, 2002.

15. Randall F, Downie RS. Palliative Care Ethics: A good companion. Oxford, Oxford University Press, 1996; 23.

16. GRACIA D. Ética de los cuidados paliativos: entre la convicción y la responsabilidad. En: Gracia D. Como arqueros al blanco. Estudios de Bioética. Ed. Triacastela 2004; 463-495.

17. Arranz P, Barbero JJ, Barreto P, Bayés R. Comunicación y Bioética en Cuidados Paliativos. En: Arranz P, Barbero JJ, Barreto P,Bayés R. Intervención emocional en cuidados paliativos. Modelos y protocolos. Ed. Ariel 2003: 141-181.

18. Roy DJ, MacDonald N. Ethical issues in Palliative Care. En. Doyle D, Hanks GWC, MacDonald N (eds): Oxford Textbook of Palliative Medicine, Oxford, Oxford University Press 1998: 99.

19. Gilligan C. In a different voice. Harvard University Press ISBN J-674-44543-9.

20. Couceiro A. Etica profesional y cuidados paliativos: en busca de la excelencia. En: Couceiro A (ed) Etica en cuidados paliativos, Triacastela 2004: 31-48.

21. GRACIA D. La deliberación moral: el método de la ética clínica. Med Clin 2001; 117: 18-23.

22. LONCÁN P et al. El plan anticipado de cuidados, un modelo de atención al final de la vida. V Congreso Mundial de Bioética. Gijon 2007.

23. http://www.secpal.com/guiasm/index.php Visitada el 5 de octubre de 2007.

24. Doty D, Walter R. Medical Futility. Clin Cardiol 2000; 23: 6-16.

25. Ética y sedación al final de la vida. Fundación Víctor Grifols. 2003.

26. Callahan D. Death and the research imperative. New Eng J Medicine 2000; 342: 654-656.
27. Ferris FD, Gómez-Batiste X, Fürst CJ, Connor S. Implementing Quality Palliative Care. J Pain Symptom Manage 2007; 33: 533-541.

28. Chapman CR, GRavin J. Suffering: the contributions of persistent pain. Lancet 1999; 353: 2233-2237.

29. Gómez SANcho M. Avances en cuidados paliativos. Las Palmas de Gran Canarias: GAFOS. 2004.

30. López Imedio E. Enfermería en cuidados paliativos. Madrid: Panamericana. 2000.

31. Alfaro-LeFevre R. Aplicación del Proceso Enfermero. Barcelona: Springer. 1998.

32. ARranz P, Barbero J, Barreto P, Bayés R. Intervención emocional en cuidados paliativos. Modelo y protocolos. Barcelona: Ariel Ciencias Médicas. 2003. ISBN: 84-3443710-4.

33. Silvestre D. "Le sida et le savoir" en Mental nº1 Junio 1995 Nouvelle École Lacanienne Págs. 141-157.

34. Maté J, GonzÁlez J, Codorniú N, Barbero E. Abordaje emocional desde un modelo interdisciplinar en cuidados plaitivos: A propósito de un caso. Libro de ponencias. III Congreso Nacional de la Sociedad Española de Psico-Oncología. 2007;pág 45

35. Barbero Gutierrez J, Bayés Sopena R, Gómez Sancho P, Torriubia Atienza P. Sufrimiento al final de la vida. En: Medicina Paliativa $n^{\circ} 2$ abril-junio vol. 142007 Madrid. Pág. 93-99.

36. Centeno C, Hernasanz S, Flores LA, Rubiales As, Lopez-LARA F. Spain: palliative care programs in Spain,2000: a national survey. J Pain Symptom Manage 2002; 24: 245-251.

37. Plan Nacional de Cuidados Paliativos. Bases para su desarrollo. 2001.

38. Plan Nacional de Cuidados Paliativos. Bases para su desarrollo. 2001.

39. Gómez-Batiste, X. El desenvolupament de les cures pal-liatives i el seu impacte en l'eficiència del sistema sanitari. Tesi Doctoral. U Barcelona. Diciembre 2003.

40. OCU. Los cuidados paliativos escasean. Encuesta OCU nº 69. Diciembre 2006.

41. ICETA M. El concepto médico de la futilidad y su aplicación clínica [tesis doctoral]. Tesis Doctoral. Pamplona: Departamento de Bioética, Universidad de Navarra, 1995.

42. Cabré Ll, Abizanda R, Baigorri F, Blanch L, CAmpos JM, Iribarren $S$ et al. Grupo de trabajo de la SEMICYUC. Código ético de la Sociedad Española de Medicina Intensiva Crítica y Unidades Coronarias (SEMICYUC). Med Intensiva 2006; 30: 1-5 
43. John Rawls. Teoría de la Justicia. Ed. Fondo de cultura económica. México DF. 1995.

44. Monzón JL, SARAlegui I, AbizAnda i CAmpos R, CABré L, Iribarren S, Martín MC, Martínez K y Grupo de Bioética de la SEMICYUC. Recomendaciones de Tratamiento al Final de la Vida del Paciente Crítico. Med Intensiva (en prensa).

45. Saralegui I, Monzón JL, Martín MC. Instrucciones Previas en Medicina Intensiva. Med Intensiva 2004; 28: 256-261.

46. Mularski, Richard A. Defining and measuring quality palliative and end-of-life care in the intensive care unit. Critical Care Medicine. Improving the Quality of End-of-Life Care in the ICU. 34 (11) Suppl: S309-S316; 2006.

47. NELSON J, DANIS M. End-of-life care in the intensive care unit: where are we now? Crit Care Med 2001; 29: N2-N9.

48. Desbiens NA, Mueller-Rizner N, Connors AF, Wenger NS, LynN J. The Symptom Burden of Seriously Ill Hospitalized Patients. J Pain Symptom Manage 1999; 17(4): 248-255.

49. Puntillo KA, Miaskowski C, Kehrle K, Stannard D, Gleeson S, Nye P. Relationship between behavioral and physiological indicators of pain, critical care patients' selfreports of pain, and opioid administration. Crit Care Med 1997; 25: 1159-1166.

50. Pardo C, Muñoz T, Chamorro C y Grupo de Trabajo de Analgesia y Sedación de la SEMICYUC. Monitorización del dolor. Recomendaciones del grupo de trabajo de analgesia y sedación de la SEMICYUC. Med Intensiva 2006; 30: 379-385.

51. Cook D, Rocker G, Giacomini M, Sinuff T, HEYLAND D. Understanding and changing attitudes toward withdrawal and withholding of life support in the intensive care unit. Crit Care Med 2006; 34: S317-S323.

52. Curtis JR, Engelberg RA. Measuring success of interventions to improve the quality of end-of-life care in the intensive care unit. Crit Care Med 2006; 34: S341-S347.

53. Azoulay E, Chevret S, Leleu G, Pochard F, Barboteau M, Adrie $\mathrm{C}$ et al. Half the families of intensive care patients experience inadequate communication with physicians. Crit Care Med 2000; 28: 3044-3049.

54. MartínEZ URIONABARRENETXEA K. La dignidad del paciente terminal. En: Ser humano, Persona y Dignidad, ed. Masiá J. Bilbao: Desclée De Brouwer, 2005; pp. 167-197.

55. LEVY M, McBRIDE D. End-of-life care in the intensive care unit: State of the art in 2006. Crit Care Med 2006; 34 (Suppl.): S306-308.
56. ANDERSHED B. Relatives in end-of-life carepart 1: a systematic review of the literature the five last years, January 1999-February 2004. J Clin Nurs 2006; 15: 1158-1169.

57. Boyle DK, Miller PA, Forbes-Thompson SA. Communication and end-of-life care in the intensive care unit: patient, family, and clinician outcomes. Crit Care Nurs Q 2005; 28: 302-316.

58. Palliative Care orders, The University Hospital of New Jersey. (http://www.theuniversity hospital.com/palliative/form.pdf) Último acceso 28/09/2007.

59. Steinhauser KE, Christakis NA, Clipp EC, McNeILly M, McInTyRe L, TulSKY JA. Factors Considered Important at the End of Life by Patients, Family, Physicians, and Other Care Providers. JAMA 2000; 284: 2476-2482.

60. CAMPBELL ML. Palliative care consultation in the intensive care unit. Critical Care Medicine. Improving the Quality of End-ofLife Care in the ICU 2006; 34(11) Suppl: S355S358; 2006.

61. DANIS M, FEDERMAN D, FINS JJ. Incorporating palliative care into critical care education: principles, challenges, and opportunities. Crit Care Med 1999; 27: 2005-2013.

62. Treece, PD, Engelberg RA, Shannon SE, NiELSEN EL, BRAUNGARDT T, RuBENFELD GD et al. Integrating palliative and critical care: Description of an intervention. Critical Care Medicine. Improving the Quality of End-ofLife Care in the ICU 2006; 34(11) Suppl: S380S387.

63. Granger CE, George C, Shelly MP. The management of bereavement on intensive care units. Intensive Care Med 1995; 21: 429436.

64. The EPEC Project (http://www.epec.net) Último acceso 26/09/2007.

65. Broggi MA, Lluviá CL, Treils J. Sufrimiento y sedación terminal. Intervención médica y buena muerte. Laboratorio de Alternativas, IBSN 84-96653-11-0. Madrid 2006. Pag 31-38.

66. Porta-Sales J, NuñEz Olarte JM et al. Aspectos éticos de la sedación en cuidados paliativos: Trabajos de Comité de Ética de la SECPAL. Med Pal 2002; 9: 41-46.

67. Hawryluck L, Harvey W, LemieuX-Charles L, SINGER P. Consensus guidelines on analgesia and sedation in dying intensive care units patients. BMC Medical Ethics 2002; 3: 3.

68. PArdo C, Muñoz T, Chamorro C y Grupo de Trabajo de Analgesia y Sedación de la SEMICYUC. Monitorización del dolor. Recomendaciones del Grupo de trabajo de 
analgesia y sedación de la SEMICYUC. Med Intensiva 2006; 30: 379-385.

69. MULARSKI RA. Pain management in the intensive care unit. Critical Care Clinics 2004; 20: 381-402.

70. Treece PD, Engelberg RA, Crowley L, Chan JD, Rubenfeld GD, Steinberg KP, Curtis JR.
Evaluation of a standarized order form for the withdrawal of life support in the intensive care unit. Crit Care Med 2004; 32: 1141-1148.

71. KIRCHHOFF KT, BECKSTRAND RL. Critical care nurses' perceptions of obstacles and helpful behaviors in providing end-of-life care to dying patients. J Critical Care 2000; 9: 96-105. 